ditions, violence was rife, and psychiatrists spent much of their time in treating casualties. Phenothiazines had little effect on aggressive psychopaths, and nursing staff experienced a great deal of anxiety in attempting to care for such people. One hesitated to admit voluntary patients suffering from mental illness to such a noisy, risk-ridden environment. The Mental Health Act of 1959 gave consultant psychiatrists the right to admit and discharge patients and as a result they discharged very many troublesome individuals so that patients with genuine mental illness could enjoy some degree of asylum in a mental hospital. It seems strange to me that Dr Bluglass, as a doctor, should suggest that a consultant who refused to admit a patient should be challenged in a test case to decide whether his refusal is "reasonable." The success of such a test case would have serious consequences for the medical profession and for patients.

In his paper Dr Bluglass makes little comment on the role of the special hospitals. $\mathrm{He}$ omits to mention that they have junior staffing problems so that much of their consultants' time is taken up with work normally done by juniors. This does not help their efficiency. The provision of special hospital places is low There are only four beds per 100000 population in England in comparison with 10 per 100000 in Scotland. Dr Bluglass does not discuss why psychiatrists are reluctant to accept patients from the special hospitals. One reason is that the Home Office continues to contro such patients and it is often impossible to persuade the Home Office to discharge them even when they are symptom-free. Few psychiatrists have the resources to care for such patients indefinitely.

I would agree with Dr Bluglass that some murderers and sexual offenders are treatable, but I would question his assertion that arsonists are in the same category. It would be valuable to have advice on how to treat this very dangerous group.

Finally, it is my view that we are attempting to solve one of the Home Office's problems with limited NHS resources. This should be made clear to the Government and money should be made available for improving psychiatric services in the prisons and for increasing the number of special hospital beds. At the same time the pay of doctors in the special hospitals should be reviewed and they should be remunerated adequately for the difficult and dangerous work they do.

JoHN T HuTCHINSON

\section{St Giles's Hospital,}

St Giles's Hospit
London SE5

\section{Septic abortion and IUCDs}

SIR,-The association of pelvic inflammatory disease (PID) with intrauterine contraceptive devices (IUCDs) is well known. In the past three years I have removed seven devices from women with clinical signs and symptoms of salpingitis. Each device was then carefully inserted into Stewart's transport medium using a sterile technique and all grew pure cultures of a particular organism. These were Escherichia coli (3), haemolytic streptococcus, Streptococcus viridans, Staphylococcus aureus, and an anaerobic bacillus, probably Clostridium welchii. Several of these women were nulliparous and their subsequent fertility has not so far been demonstrated as far as I know. The report by $\mathrm{Mr}$ R A Sparks and Mr A T
Letchworth (25 February, p 482) of a septic abortion in an IUCD user identified three organisms ( $E$ coli, a streptococcus, and a staphylococcus) although only the coliform was found in the blood.

During the same period I have seen at least six cases of PID due to gonococcus associated with IUCDs, although I have no statistical evidence to back up my clinical impression that this complication is more likely in the presence of a foreign body in the uterus. None of the devices in either group was a Dalkon Shield, although four years ago one of the only two cases of septic mid-trimester abortion associated with this device discovered in the UK was in a patient of the Glasgow Family Planning Service (Domiciliary). The infection was gonorrhoea and the girl, who was retarded and homeless, was found by the police bleeding heavily and severely ill in a condemned tenement.

It has been the policy of this centre to remove all IUCDs as early in a confirmed pregnancy as possible since Cates et al $^{1}$ showed that the chances of a late spontaneous miscarriage were much greater if the device was left in situ. Vessey's work also demonstrated that the chances of a pregnancy going to term with the device in place were only one in two.

It is surely preferable to take the very slight risk of causing an immediate miscarriage by removing the device in the first three months, when if it occurs it is physically and mentally less traumatic and medically more easily supervised, than leaving it in and risking a spontaneous abortion at 16 weeks or later. The latter may possibly be associated with sepsis as US reports and now the report of $\mathrm{Mr}$ Sparks and Mr Letchworth show, and the patient may well be on holiday abroad and not necessarily within reach of free or effective medical care.

Ten years ago a patient in whom I had fitted a Lippes Loop became pregnant and, in spite of repeated threatened miscarriages and a severe antepartum haemorrhage, produced a live premature baby at 30 weeks. This child is permanently brain damaged. This and the possible death of the mother due to uncontrollable sepsis are very high prices to pay for timorousness on the part of the doctor who diagnoses the pregnancy. The device should be removed as soon as possible and not left until the patient reaches the obstetrician at the antenatal clinic, which in this city is often not until well past 12 weeks.

I would also suggest, with reference to the report by Mr Sparks and Mr Letchworth, that a high vaginal swab is quite useless in the diagnosis of PID and I cannot see that cutting the threads flush with the external os would make any difference whatever.

E S B WILSON

Family Planning Association,

Glasgow Family Planning Centre, Cates, W, jun, et al, New England fournal of Medicine,
1976, 295, 1155.

SIR,-I was interested to read the report by Mr R A Sparks and Mr A T Latchworth (25 February, $p$ 481) of an infected abortion associated with the use of a Gravigard intrauterine contraceptive device (IUCD). The device was removed at 16 weeks when the patient was admitted for the second time. Such a removal can be performed only if the thread has been left extraordinarily long or when the IUCD is lying in the cervical canal. Under normal circumstances by 16 weeks the thread will have disappeared.

It seems unlikely that in this case the thread had been left much too long and far more probable that the device was removed from the upper part of the cervix, which would explain why it had failed as a contraceptive. An alternative suggestion is that a normally placed IUCD might have been extruded into the cervix at the time of the patient's first bleeding and thus allowed infection to contaminate some residual blood clot. It would be interesting to know the time interval in this case between fitting the IUCD and the pregnancy and also whether the tail of the IUCD proved to be unduly long.

I agree that it is always best to remove any IUCD associated with pregnancy provided the tail can still be seen. The risks of removal have been, in my experience, considerably less than those of leaving it inside.

\section{Wilfrid Mills}

Queen Elizabeth Maternity Hospital,
Birmingham

\section{Pelvic actinomycosis associated with IUCD}

SIR,-We were interested to read the report by Dr D W Purdie and others (26 November 1977, p 1392) of tubo-ovarian actinomycosis in the presence of a plastic intrauterine contraceptive device (IUCD). We believe that this type of actinomycosis is more common than has been previously suspected. New cases have been seen recently in several hospitals in England: one of us (ACB) has seen two cases in Sheffield, we have learnt of at least four cases at other London teaching hospitals, and another has been notified recently in the Communicable Disease Report. ${ }^{2}$ We would like to report a further case seen at our hospital in which serological tests for actinomycosis were positive and suggest that such tests might be of value for diagnosis and management of this infection.

A 38-year-old Ethiopian woman, para $1+2$ (terminations in 1963 and 1968), had used an IUCD since 1968 with two-yearly changes. The final device, an Italian plastic coil with no metal, was removed in October 1977. She was admitted to hospital in November having suffered lower backache and pain in the right leg for eight months, intermittent pain in the right iliac fossa for five months, and more recently intermittent haematuria and malaise. Her periods were regular and there was no vaginal discharge. A firm, tender mass was palpable in the right iliac fossa, continuing into the pelvis. Haematological results included haemoglobin $11.4 \mathrm{~g} / \mathrm{dl}$ and white cell count $15.9 \times 10^{9} / 1$ $\left(15900 / \mathrm{mm}^{3}\right)$. At laparotomy the mass was found to infiltrate the wall of the pelvis on the right with an enlarged adherent right ovary but normal uterus and tubes. The terminal ileum was adherent to the mass at the pelvic brim and the right ureter was displaced and narrowed. It was not clear whether the lesion had spread from the gut or from the genital tract. Ovarian biopsy was performed, which revealed typical actinomycotic "sulphur granules," and Actinomyces israelii was isolated from the specimen. Oral and cervical cultures did not yield the organism. The patient's serum contained precipitins to standard $A$ israelii cytoplasmic antigens, and circulating C1-binding immune complexes containing IgG and IgA were demonstrated. The patient was treated with a combination of penicillin and fusidic acid at first and is now continuing with penicillin alone; there has been symptomatic improvement.

There has been interest in the use of serology for the diagnosis of actinomycosis for years 
and recently Holmberg et al have reported developments which have improved the specificity of the tests. ${ }^{3}$ We tested our patient's serum by counterimmunoelectrophoresis using a pooled cytoplasmic extract from strains of $A$ israelii kindly given to us by Dr Holmberg. Serological tests might be helpful for diagnosis as specimens are not easily collected for culture or histological examination in pelvic infections. The presence of a slow-growing anaerobe could easily be missed if there were a mixed growth of organisms on culture, and short courses of antibiotics might prevent cultural diagnosis. Serological testing of patients with long-standing pelvic inflammatory disease, particularly when associated with an IUCD, could be useful for detecting actinomycosis which might otherwise be missed. Circulating immune complexes were demonstrated in our case; we suggest that the disappearance of such complexes may be a useful marker for adequacy of treatment.

We wish to thank Mr A C Fraser for permission to publish details of his patient and $\mathrm{Mr} M$ Savage and Professor J Mowbray for performing the serological tests.

Michael BarNhaM ANTHONY C BURTON Paul Copland

Departments of Microbiology, St Mynaecology, and Urology,

St Mary's Hos

1 E Houang, personal communication.

Communicable Disease Surveillance Centre of the

Public Health Laboratory Service, Communicable
Disease Report, 1978, No 5.
${ }^{3}$ Holmberg, K, Nord, C E, and Wadstrom, T, Infection and Immunity, 1975, 12, 387 and 398.

\section{Is pancreatic isotope scanning worth} while?

SIR,-The short report by Dr Peter Cotton and his associates on isotope scanning of the pancreas (4 February, p 282) prompts us to record our recent results with this technique and explore some of the possible reasons why our experience differs.

We have previously published a study in 102 patients examined with a rectilinear scanner. ${ }^{1}$ We now report the results in 200 patients examined by General Electric Radicamera with a Med II system. Scans were interpreted by three observers with experience of the technique but without knowledge of the precise clinical situation. The results are shown in the table below.

With experience and superior equipment the incidence of technically unsatisfactory scans is now negligible. However, compared with our previous study the false-positive rate is higher $-36 \%$ compared with $30 \%$-a trend which reflects an acknowledged bias towards reporting as abnormal a scan which is not unequivocally normal. Such a policy is of practical value when, as in this department, isotope scanning is used primarily as a screening test to select those patients who require more detailed investigation for, in our experience and as others report, a normal scan virtually excludes chronic pancreatitis and cancer. ${ }^{1-3}$

The high rate of "equivocal" reports, $20 \%$, reported by Dr Cotton and his colleagues, may partly be conditioned by the apparatus used and influences the results in both normal and disease groups. Though we have no experience with the Elscint whole-body scanner, we abandoned the use of the Nuclear Enterprises gamma camera for pancreatic work after a few months' use in favour of the old rectilinear scanner, which provided more satisfactory imaging. We find it difficult to account for their low number of abnormal scans in patients with chronic pancreatitis and cancer $(59 \%)$. Even if the equivocal reports are counted as abnormal the percentage rises only to 79 . However, the results in relapsing pancreatitis are explicable since in relapsing acute pancreatitis the timing of the scan is all-important; total recovery of function can be anticipated within 6-8 weeks of the acute relapse. Dr Cotton and his colleagues do not distinguish between relapsing chronic and relapsing acute pancreatitis. The disadvantage of isotope scanning other than the high false-positive rate in subjects with a normal pancreas is that the scan cannot be repeated to follow functional progress in the individual patient within 4-6 months.

Finally, in our experience ultrasonography is not an ideal primary screening test since it depends even more than isotope scanning on the individual operator's technical expertise. In agreement with Doust and Pearce ${ }^{5}$ we have observed that in some $50 \%$ of patients with quiescent chronic pancreatitis the ultrasonic scan is normal. In these isotope scanning is abnormal in $96 \%$ of cases.

H J TESTA

Mary C Prescott

J BRAGANZA HENRY T HOWAT

Department of Nuclear Medicine and

University Department of

Gastroenterology,

Manchester

'Braganza, J M, et al, Gut, 1973, 14, 383.
2 Mitchell, C J, et al, British Medical fournal, 1976, 2, Mitche

1307. J E, Maze, M, and Mitchell, C J, British
${ }^{3}$ Anew, J E Aournal of Radiology, 1976, 49, 979.

Russell, J G B, et al, Gut, 1977, 18, A418.

'Doust, B, and Pearce, J, Radiology, 1976, 120, 653.

\section{Coronary heart disease, age, and sex}

SiR,-Drs R F Heller and H S Jacobs (25 February, p 472) suggest that testosterone may be responsible for the extra risk of coronary heart disease (CHD) that men before the age of 50 years encounter as compared with women of the same age; but "the mechanism of any risk it might produce is not clear."

More than 20 years ago $I^{1}$ discussed the possible relation of a relative deficiency of essential fatty acids (EFA) to atherosclerosis

Scan reports on 200 patients

\begin{tabular}{|c|c|c|c|}
\hline & \multirow{2}{*}{ No of patients } & \multicolumn{2}{|c|}{ Scan report } \\
\hline & & Normal & Abnormal \\
\hline 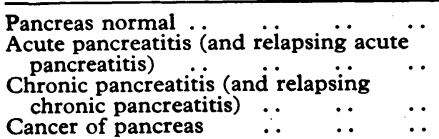 & $\left.\begin{array}{l}137 \\
15 \\
25 \\
23\end{array}\right\} 48$ & 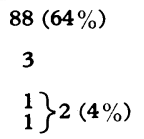 & $\begin{array}{l}\left.\begin{array}{l}49(36 \%) \\
12 \\
24 \\
22\end{array}\right\} 46(96 \%)\end{array}$ \\
\hline
\end{tabular}

and $\mathrm{CHD}$ and pointed out that as "the requirement of male animals for EFA is at least five times that of females," if we argued from lower animals to man "deficiency would be likely to be at least five times commoner in males than in females." The ratio of the male to female mortality rates for CHD (ICD 410-414) in England and Wales in 1973 was $6 \cdot 1$ at age $35-44$ years and $5 \cdot 5$ at age 45 to 54 years. If the animal is castrated the requirement of the male is the same as that of the female.

Since prostaglandins are formed in the body only from EFA and since human males excrete roughly five times as much prostaglandin metabolites as do females ${ }^{2}$ it might be supposed that the increased male requirement of EFA is caused by increased synthesis of prostaglandins. But this synthesis accounts for only a very small proportion of the daily requirement of EFA. For a reason that is obscure male animals become much more depleted than do females on a diet low in EFA. ${ }^{3}$

Hugh Sinclair

\section{International Institute of}

Human Nutrition,

Sutton Courtenay, Oxon

1 Sinclair, H M, Lancet, 1956, 1, 381.

Samuelsson, B, et al, Annals of the New York Academy of Sciences, 1971, 180, 138 . Academy of Sclences, $1971,180,138$.
Research, 1965, 6, 287 .

\section{Diet and heart: the importance of metals}

SIR,-In their very interesting prospective study on diet and the heart (19 November, p 1307) Professor J N Morris and his colleagues mention the possibility that their results might be accounted for by deficiencies in the diet of those with low energy intake. There may well be a reduced intake of mineral salts in those with low energy diets and there is now evidence that sudden death from ischaemic heart disease is associated with decreased concentrations of some metals in the heart muscle.

Decreased concentrations of magnesium in the hearts of those dying suddenly from ischaemic heart disease have been reported in several studies, ${ }^{1-3}$ and Seelig ${ }^{4}$ has estimated that Western diets provide insufficient magnesium to maintain magnesium balance in men. There is a significant decrease in the concentration of magnesium in the heart muscle of normal men compared with normal women, ${ }^{5}$ which corresponds with the increased incidence of ischaemic heart disease in men.

In addition there are decreased concentrations of potassium and iron ${ }^{6}$ and an increased concentration of calcium $^{36}$ in the heart muscle of subjects dying suddenly from ischaemic heart disease. The increase in calcium, as suggested by Anderson et $a l,{ }^{3}$ is probably secondary to the decreased magnesium concentration. These results therefore suggest that those dying suddenly from ischaemic heart disease are relatively deficient in magnesium, potassium, and iron. Deficiencies of these same metals have been identified as contributing to the clinical problems in kwashiorkor and we have suggested that "empty calorie malnutrition"diets adequate in calories but low in essential metals-may affect the heart both in kwashiorkor and in ischaemic heart disease. ${ }^{6}$

Published figures show ${ }^{7}$ that brown bread contains $30 \%$ more iron, twice as much potassium, and three times as much magnesium as white bread. The group 